\title{
Effect of casing yield stress on bomb blast impulse
}

\author{
M.D. Hutchinson \\ AWE Aldermaston, Reading RG7 4PR, UK
}

\begin{abstract}
An equation to predict blast effects from cased charges was first proposed by U. Fano in 1944 and revised by E.M. Fisher in 1953 [1]. Fisher's revision provides much better matches to available blast impulse data, but still requires empirical parameter adjustments. A new derivation [2], based on the work of R.W. Gurney [3] and G.I. Taylor [4], has resulted in an equation which nearly matches experimental data. This new analytical model is also capable of being extended, through the incorporation of additional physics, such as the effects of early case fracture, finite casing thickness, casing metal strain energy dissipation, explosive gas escape through casing fractures and the comparative dynamics of blast wave and metal fragment impacts. This paper will focus on the choice of relevant case fracture strain criterion, as it will be shown that this allows the explicit inclusion of the dynamic properties of the explosive and casing metal. It will include a review and critique of the most significant earlier work on this topic, contained in a paper by Hoggatt and Recht [5]. Using this extended analytical model, good matches can readily be made to available free-field blast impulse data, without any empirical adjustments being needed. Further work will be required to apply this model to aluminised and other highly oxygen-deficient explosives.
\end{abstract}

\section{Blast impulse equations}

The equation derived in [2] for the blast impulse $I$ from a cased charge as a fraction of the impulse $I_{0}$ from the same charge without a casing is:

$$
\frac{I}{I_{0}}=\sqrt{\frac{C}{C+2 M}}
$$

This equation applies where the casing metal is very ductile and therefore expands to a radius at which the internal driving pressure of the explosive gases is negligible. It also applies only to explosive compositions that are neither aluminised nor otherwise highly oxygen deficient, since these generate additional blast energy through exothermic reactions with the surrounding air (i.e. after-burn).

However, many real bomb casings are made from metals with significant yield strength and these will fracture at expansion radii where internal driving pressure of the explosive gases is significant and the simple energy balance between gases and casing fragments predicted by Gurney [3] has not been reached.

\section{Casing fracture criteria}

It has been pointed out by G.I. Taylor [4] that the internal driving pressure exerted upon the casing metal by the gaseous products, while it eventually strains the casing metal towards fracture, initially suppresses fracture. The casing material is initially compressed between the gas pressure acting on its inside surface and its own inertia. The casing metal shears in compression, both losing thickness and gaining diameter and surface area in the process.

Taylor also pointed out that the compressive stress in the casing falls in value from the instantaneous gas pressure at its inside surface, as expressed by the following equation

$$
P=P_{0}\left(r / r_{0}\right)^{-2 \gamma}
$$

for a perfect gas, to near zero (i.e. just the pressure of any surrounding air) at its outside surface.

This means, as illustrated in figure 1 , that as the internal gas pressure $P$ reduces adiabatically with advancing casing expansion, a release wave propagates inwards from the casing outer surface. Behind this wave, the casing material can fracture. Ahead of this wave, the material can still yield in compressive shear.

According to Taylor [4], the Tresca criterion for shear failure applies at the point where this effective release wave reaches the casing inner surface, and this defines the critical stress condition at which the casing metal is obliged instead to fracture and then expand as an envelope of discrete fragments.

The choice of criterion to define the critical stress condition for through-casing fracture was subsequently reviewed by Hoggatt and Recht [5] and they proposed a different failure criterion, i.e. that the stress components, normal to a shear plane in the casing metal, should sum to zero. However, this paper calls this new approach by Hoggatt and Recht into question and argues for the original Taylor/Tresca criterion, based on the following two points:

Firstly, the Tresca criterion used by Taylor is that most relevant on the prediction of shear yield in response to biaxial stresses.

Secondly, Hoggatt \& Recht's criterion predicts failure at higher strain than does the Taylor/Tresca criterion, which would thus allow fracture first.

Hoggatt and Recht themselves found evidence of shear strain localisation into adiabatic shear bands. Considering the weakened condition of casing material within these bands, a crack should propagate inwards along such a band, as soon as the compressive shear could no longer be maintained. Thus it is hard to see why the stress components, normal to a shear plane in the casing metal, should first have to sum to zero, as in Hoggatt \& Recht's criterion.

Nonetheless, the internal dynamics of expanded metal casings have a significant impact on the shape, size 


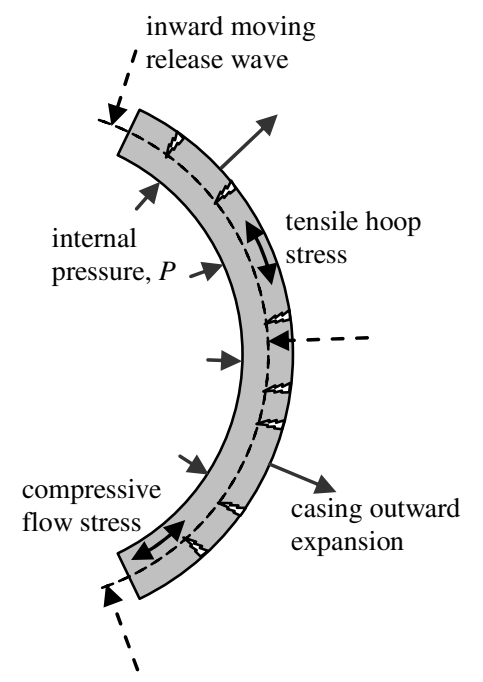

Fig. 1. Illustrating the mechanism identified by Taylor.

distribution and initial velocity of the projected casing fragments and, for the purposes of this paper, on blast impulse. The mathematical methods of Hoggatt and Recht provide a valuable insight into those internal dynamics and will therefore be reviewed here.

\section{Radial dependency of compressive stress}

Hoggatt and Recht approach the solution for the dynamic radius within the casing thickness, at which the critical stress condition exists, via a number of stages, the last of which is numerical rather than analytical. Firstly, an equation of motion is derived for the wall of a cylindrical bomb. Then, based on casing metal volume conservation, the dynamic radius $a$ of any point within the casing thickness can be derived, relative to its initial radius, $a_{0}$ :

$$
a / a_{0}=\left(r^{2} / a_{0}^{2}-r_{0}^{2} / a_{0}^{2}+1\right)^{1 / 2}
$$

from this can be derived the following expression for the radial pressure $p_{a}$ in the wall, at any radius a:

$$
p_{a}=\left[P^{r} /\left(r^{2}+a_{0}^{2}-r_{0}^{2}\right)^{1 / 2}\right] \cdot\left[\left(R_{0}^{2}-a_{0}^{2}\right) /\left(R_{0}^{2}-r_{0}^{2}\right)\right]
$$

In (4), $r$ is the radius of the inner surface, $R$ the outer surface and $a$ an arbitrary point through the thickness of the casing, $r<a<R$. Subscripted terms refer to the radius of these three points at time $t=0$. Equation (2) for the fall in gas pressure with casing expansion is derived, but is based on the assumption that the initial mean gas pressure will be the Chapman-Jouget pressure, $P_{C J}$. In reality, $P_{C J}$ is only reached just behind a detonation wave and the true initial mean pressure $P_{0} \approx 0.42 P_{C J}$. An empirical relation given between the compressive plastic natural strain and the compressive uniaxial stress, $p_{a e}$ :

$$
\varepsilon_{p}=-\left(p_{a e} / k\right)^{1 / n}
$$

where $\mathrm{k}$ is a strength coefficient and $\mathrm{n}$, a work hardening component. A Von Mises (three-dimensional) description is given of the compressive uniaxial stress, where $\sigma_{\theta}$ and $\sigma_{Z}$ are the hoop and axial stress components:

$p_{a e}=-\left(\frac{1}{\sqrt{2}}\right)\left[\left(-p_{a}-\sigma_{\theta}\right)^{2}+\left(\sigma_{\theta}-\sigma_{Z}\right)^{2}+\left(\sigma_{Z}+p_{a}\right)^{2}\right]^{1 / 2}$

Eliminating the underlying elastic strains, as defined by the tri-axial stress-strain equations that incorporate Young's modulus, $Y$, and Poisson's ratio, $v$, Hoggatt and Recht derived values of $a_{p}$, the purely plastically expanded radius of a differential element of casing originally situated at radius $a_{0}$ :

$$
a_{p}=a /\left[1-\left(v^{2}+v\right) p_{a} / Y\right]
$$

Equation (7) is their solution for $\sigma_{\theta}=0$, the Taylor/Tresca criterion, rather than Hoggatt \& Recht's own failure criterion. In (7), $a$ is the actual expanded radius of the casing thickness element, including the elastic strain. The final analytical steps are to derive the corresponding value of the equivalent compressive stress:

$$
p_{a e}=p_{a}\left(1-v+v^{2}\right)^{1 / 2}
$$

and, from (3), (4) and (5) the failure strain at any expanded radius a within the casing thickness:

$$
a / a_{0}=\left[1+\left(p_{a} / Y\right)\left(v^{2}+v\right)\right] \exp \left(\left(p_{a} / k\right)\left(1-v+v^{2}\right)^{1 / 2}\right)^{1 / n}
$$

Further details can be found in Hoggatt \& Recht's own paper [5], however, the above analytical derivation does not lead to analytical solutions. Values for $p_{a}$ in (5) must be found by first finding the value of $a_{0}$ at a selected value of expansion radius $r$. Based on equation (3) for the dynamic radius, $a$ of an element within the casing, and knowing the instantaneous mean gas pressure from equation (2) equation (4) therefore becomes:

$$
p_{a}=P_{0}\left(\frac{r}{r_{0}}\right)^{-2 \gamma}\left[\frac{r}{\left(r^{2}+a_{0}^{2}-r_{0}^{2}\right)^{1 / 2}}\right]\left[\left(R_{0}^{2}-a_{0}^{2}\right) /\left(R_{0}^{2}-r_{0}^{2}\right)\right]
$$

Hoggatt and Recht were then able to find, by iteration, $a / a_{0}$ values that obeyed both the geometric requirement in (10) and the stress condition $\sigma_{\theta}=0$.

As a check on this rather complex iterative method of deriving failure strain values, it can be shown that the point at which the failure condition appears at the inner surface can be found more simply by solving only for the inner surface of the casing, i.e. when $a_{0}=r_{0}$ and $a=r$. Based on equation (9), and substituting for the value of $p_{a}=P$ at inner radius $r$ from equation (2), one can obtain the following part-logarithmic, part-polynomial equation for the reciprocal of failure radius $r_{f}$ :

$$
\begin{aligned}
& \ln \left(\frac{r_{0}}{r_{f}}\right)+\ln \left[1+\left(\frac{P_{0}}{Y}\right)\left(\frac{r_{f}}{r_{0}}\right)^{2 \gamma}\left(v^{2}+v\right)\right] \\
& +\left(\left(\frac{P_{0}}{k}\right)\left(\frac{r_{f}}{r_{0}}\right)^{2 \gamma}\left(1-v+v^{2}\right)^{1 / 2}\right)^{1 / n}=0
\end{aligned}
$$




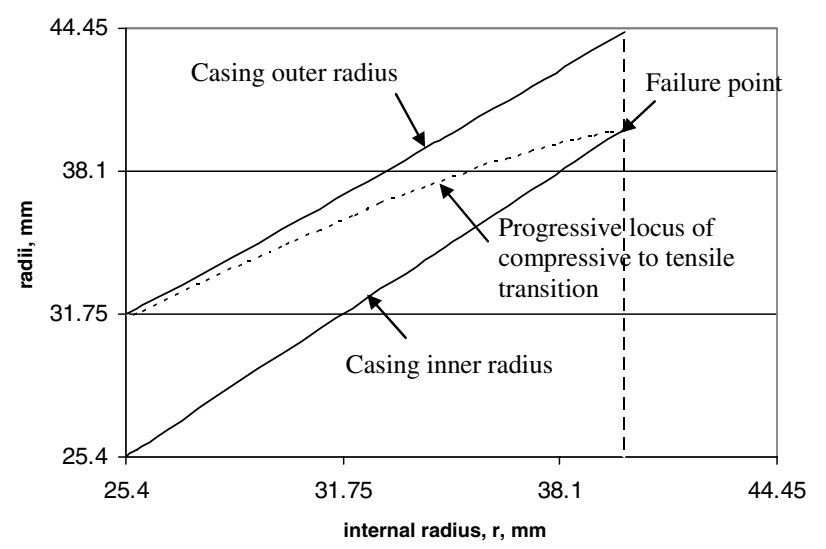

Fig. 2. Progress of the compressive-tensile stress boundary though the casing metal.

However, even this equation does not have a straightforward analytical solution. Remaining therefore with iterative methods, but using equation (11) as a check on the end values, it is possible to match very closely the values of $a$ and $r$ at which dynamic failure should occur with those obtained by Hoggatt \& Recht [5] for a worked example from Taylor [4].

\section{Example from Taylor}

In order to demonstrate their iterative method, Hoggatt and Recht used the example of a steel cased bomb given by Taylor, together with textbook values for $Y, k, v$ and $n$ for the mild steel tube of internal radius $\operatorname{lin}(0.0254 \mathrm{~m})$ and external radius 1.25 in $(0.0318 \mathrm{~m})$, packed with reduced density $(1.5 \mathrm{~g} / \mathrm{cc})$ RDX explosive. Of significance here is that both Hoggatt and Recht and Taylor made the error of using a $P_{C J}$ value of $20.9 \mathrm{GPa}$ for the initial pressure $P_{0}$ when the value used should have been $8.8 \mathrm{GPa}$.

The values of $a_{0}$ were stepped in intervals of $0.01 \mathrm{in}$, $0.254 \mathrm{~mm}$ from $r_{0}$ (= $\left.1.0 \mathrm{in}, 25.4 \mathrm{~mm}\right)$ to $R_{0}(=1.25 \mathrm{in}$, $31.8 \mathrm{~mm}$ ). The values of $r$ for each value of $a_{0}$ were then adjusted by trial and error to bring those values of $a / a_{0}$ into line with the values of $a / a_{0}$ derived for each stress condition $p_{a}$. A simple macro was written to use the goal seek function in MS-Excel to look for a value of $r$ for which the two values of $a / a_{0}$ had a ratio of 1.0 and it was found that this gave the necessary values for the required curves to $< \pm 0.1 \%$.

In figure 2, the straight black lines show the changing casing inner and outer radii with increasing inner radius. The casing material is located between these two lines. The dotted curve, based on the above up-to-date version of Hoggatt and Recht's iterative method, plots the loci of radii a within the dynamic casing thickness at which, according to Taylor, the material is in transition from compressive to tensile stress.

This curve effectively shows the progress of a wave, starting from the outer casing surface, which allows fracture when it reaches the dynamic inner surface (lower straight line). When the value of $a$, which is increasing more slowly than $r$, is caught up with at $r=r_{f}$, all the casing material is in tension and through-fracture can occur.

Working through method of Hoggatt and Recht, while retaining the Taylor/Tresca failure criterion, provides the necessary underpinning in the form of a stress-based fracture methodology to predict modified blast impulse in the presence of strong casings.

\section{Blast impulse modified for casing yield stress}

In a further paper [6] it will be shown that the work energy $E C$ remaining with the explosive gases at the radius of casing fracture can be expressed by the following modified version of equation (1):

$$
\frac{E_{C}}{E C}=\frac{C+2 M\left(r_{0} / r_{f}\right)^{2(\gamma-1)}}{C+2 M}
$$

where $E$ is the total work energy (i.e. Gurney energy) available per unit charge mass, $C$ is the mass of explosive and $M$ the mass of casing and $r_{f}$ the casing inner radius at fracture. Applying Taylor's fracture criterion, the gas pressure $P$ within the casing can firstly be related to the radius $r_{f}$ to which the casing has expanded from its initial radius $r_{0}$ and to the initial gas internal pressure $P_{0}$, and secondly equated to the metal yield stress, $\sigma_{y}$ :

$$
P=P_{0}\left(\frac{r_{0}}{r_{f}}\right)^{2 \gamma}=\sigma_{y}
$$

Therefore, rearranging the right hand equation in (13), at casing failure:

$$
\frac{r_{0}}{r_{f}}=\left(\frac{\sigma_{y}}{P_{0}}\right)^{1 / 2 \gamma}
$$

Using equation (14) to substitute for $r / r_{0}$ in (12), we now obtain:

$$
\frac{E_{C}}{E C}=\frac{C+2 M\left(\sigma_{y} / P_{0}\right)^{(\gamma-1) / \gamma}}{C+2 M}
$$

While gas kinetic energy and gas momentum are not simply related, due to the distribution of velocities within the gas, the same proportionality should hold for both bare and cased charges of the same geometry. Therefore, by taking the square root of the right-hand side of (15), we can obtain the blast impulse $I$ of the cased charge as a fraction of the impulse $I_{0}$ from the same charge without a casing:

$$
\frac{I}{I_{0}}=\sqrt{\frac{C+2 M\left(\sigma_{y} / P_{0}\right)^{(\gamma-1) / \gamma}}{C+2 M}}
$$

Thus we have a new equation for the blast impulse from a cased charge, which only requires us to know the casing/charge mass ratio, the explosive properties and the casing metal yield stress. 


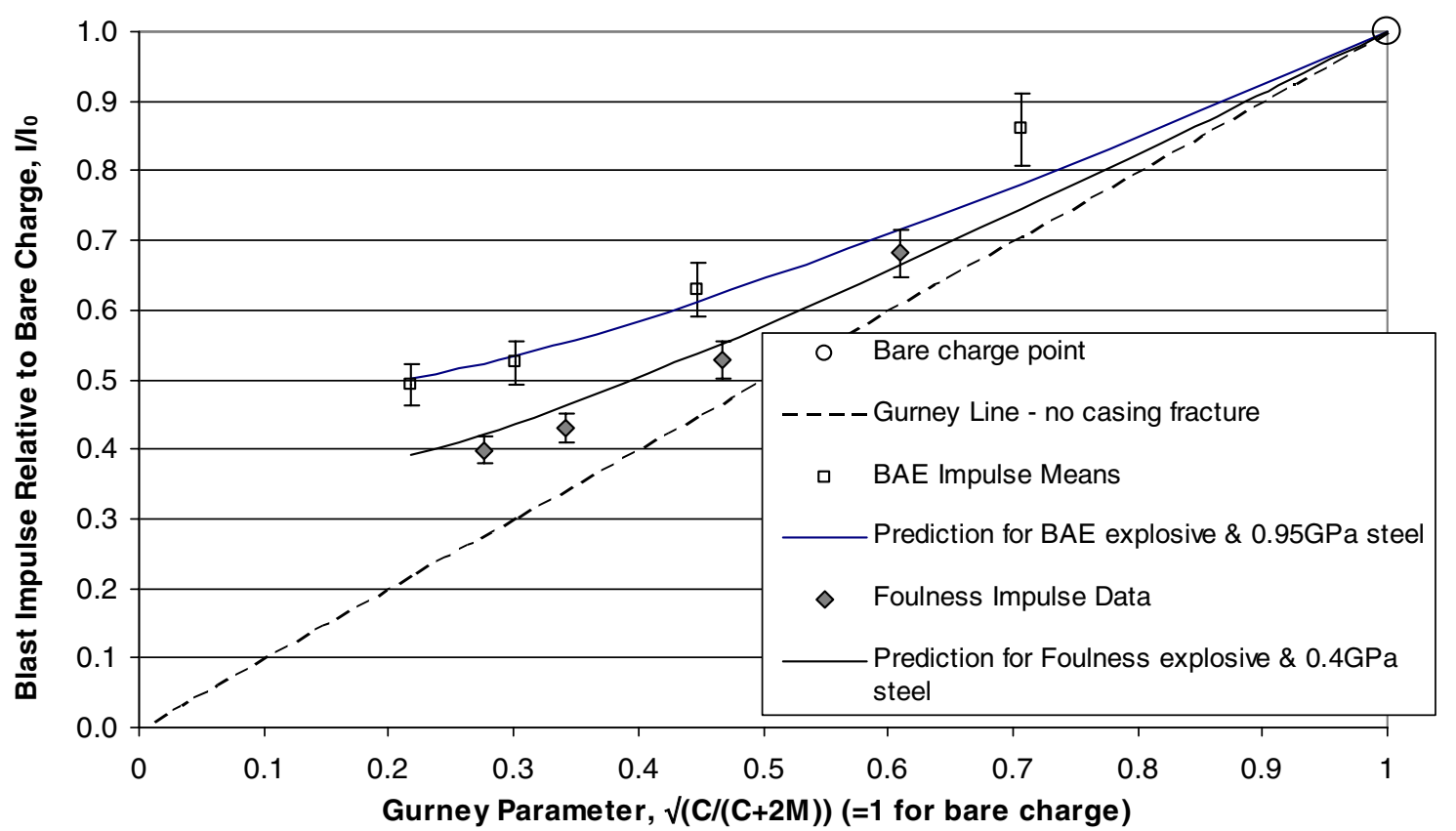

Fig. 3. Plot of predicted (equation 16) and experimental relative blast impulses against the function $\sqrt{ }(C /(C+2 M))$ of the charge and casing masses. Points for thicker casings are towards the left.

\section{Experimental comparison}

Figure 3, a plot in Gurney parameter space shows good comparisons between the predictions of equation (16) and blast impulse data from two independent sets of experiments, one from an unpublished report by Bishop and James at AWE Foulness in 1970 and another data set published much more recently by Flynn and Wharton [7].

Both sets of data were for cylindrical charges, i.e. steel tubes of varying steel thickness and mass filled with nonaluminised explosives. In all instances, side-on pressure gauges were ranged at intervals along a radius struck from the mid-point charge axis. Dynamic pressure readings have been time integrated to provide relative side-on impulse values.

The diagonal straight dotted line is the prediction of equation (1). It is referred to here as the 'Gurney Line' because it represents the condition where the final energy balance between casing fragments and explosive gases is that postulated by Gurney [3]. The point in the top right hand corner is that for a bare charge, in the bottom left hand corner is the point for an infinitely heavy casing.

The smooth, shallow curves are the predictions of equation (16), both for the stronger steel $(0.96 \mathrm{GPa})$ and more powerful explosive used in the BAE Systems experiments, and the milder steel $(0.4 \mathrm{GPa})$ and less powerful explosive used in the AWE Foulness experiments.

\section{Conclusions}

Regarding the internal dynamics of the casing metal, the analytical method of Hoggatt and Recht [5] provides a valuable expansion and conformation of the methodology first set out by Taylor, but the Tresca fracture criterion adopted by Taylor [4] should be adhered to. Significant misunderstandings regarding the inertia and initial pressure of the explosive gases exist in these previous papers and should be noted.

The Tresca/Taylor criterion can be used as a basis on which to derive an equation (16) for cased charge relative blast impulse which allows for the casing and explosive dynamic properties. The available experimental data validate the predictions of this equation, regarding the effect of different charge compositions and steel casing yield stresses. It is thus concluded that this equation ought to replace any empirical equations currently in use to predict the relative blast impulse of cased charges. Further work will be required to apply this model to aluminised and other highly oxygen-deficient explosives.

\section{References}

1. E.M. Fisher, U.S. Naval Ordnance Laboratory, NAVORD Report 2753 (1953)

2. M.D. Hutchinson, Propellants Explos. Pyrotech. 36, 310-313 (2011)

3. R.W. Gurney, U.S. Ballistics Research Laboratories BRL Report 405 (1943)

4. G.I. Taylor, Scientific Papers of G.I. Taylor, (Cambridge University Press, 3, 387, 1963)

5. C.R. Hoggatt, R.F. Recht, J. Applied Physics, 39, 3 (1968)

6. M.D. Hutchinson, Propellants Explos. Pyrotech. (to be published)

7. J. Dunnett, D. Flynn, P. Wharton, IMEMTS Proceedings (2006) 